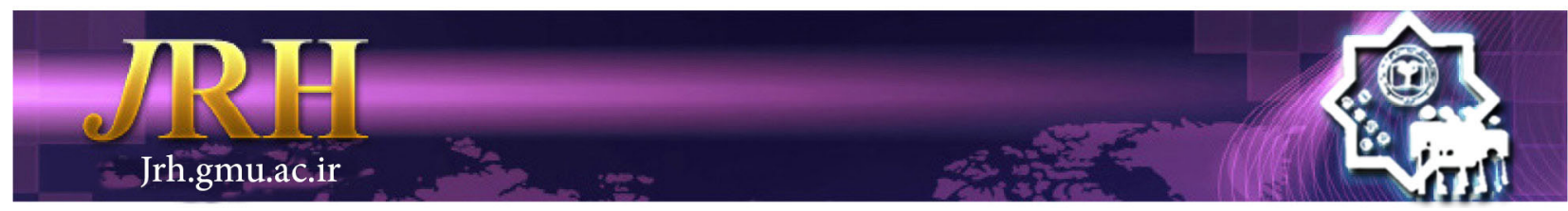

\title{
Comparison of the effectiveness of training of reality and spirituality on psychological well-being among students
}

Fatemeh Nemati Sogolitappeh ${ }^{1}$

\author{
Journal of Research \& Health \\ Social Development \& Health Promotion \\ Research Center \\ Vol. 8, No.5, Sep \& Oct 2018 \\ Pages: $473-482$ \\ DOI: $10.29252 / j r h .8 .5 .473$ \\ Original Article
}

1. Correspondence to: Department of Psychology, University of Tabriz, Tabriz, Iran

Email: f.nemati84@gmail.com

Received: 5 Nov 2017

Accepted: 20 May 2018

How to cite this article: Nemati Sogolitappeh F. Comparison of the effectiveness of training of reality and spirituality on psychological well-being among students. $J$ Research \& Health2018; 8(5): 473- 482.

\begin{abstract}
Positive psychology is one of the issues which have attracted a lot of attention in recent decades. The current research was conducted with the aim of comparing the effectiveness of training of reality and the spirituality on promoting psychological well-being in students. The method of this research was semi-experimental with experimental and control groups. In this study 45 university students were selected by sampling method as sample size and then the participants were randomly divided into three groups (two experimental and one control groups) which each group had 15 participants. A test group received nine 90-minute sessions of group-based reality training; the second group received nine sessions of two-hour spirituality training in group way, but the control group did not receive any intervention. Ryff's Psychological Well-Being Scales was used to collect information. The results showed that spiritual education has a higher effect on the education of reality in promoting the level of psychological well-being in students. Thus, it can be concluded that reality is influential in promoting the psychological well-being of the students and also the spiritual education and reality education significantly affect the level of psychological well-being in students, but spirituality is more effective than reality.
\end{abstract}

Keywords: Psychological Well-being, Reality Training, Student

\section{Introduction}

Positive psychology is one of the issues which have attracted a lot of attention in recent decades. Positive psychology is the scientific approach of the optimal human performance and focuses on how human life flourishes and how human can reach its abilities [1]. Wellbeing can be classified into two axes of subjectmatter and psychological well-being; the subject's well-being has emotional (positive and negative affection balance) and psychological components (individual's assessment for life satisfaction); in fact, the subject of well-being is a kind of happiness and the psychological well-being is an employment with the ontological challenges of life [2]. In the past decade, Ryff et al presented a pattern of psychological wellbeing or positive mental health. Based on the Ryff's model, psychological well-being consists of six factors; self-acceptance (having a positive attitude and self-ability), positive relationship with others (establishing warm and intimate interactions with others and the ability of empathy), autonomy (independence 
feeling and ability to withstand social pressures), purposeful life (having purpose in life and meaning about it), personal growth (continuous growth feeling) and environmental mastery (individual ability to manage the environment) [3]. The Ryff pattern has been widespread around the world, and Ryff presented this pattern on the basis of a review of mental health literature and stated that the components of the model are positive mental health standards, and these dimensions help to measure the level of well-being and positive person's performance. Psychological wellbeing has been defined as the growth of the real talent of each individual [4]. Psychological well-being includes positive feelings and sense of general satisfaction with life which includes self and others in different areas of the family, occupation and so on. Therefore, people with high self-esteem experience more positive emotions and have positive assessment of events and happenings around them. On the one hand, individuals with low-welfare evaluate their adverse events and their situations and experience more negative emotions such as anxiety, depression and anger [5]. Nowadays issues such as psychology, religion, spirituality and so on have been the subject of research and critique of psychology scholars for many years and institutions, associations, research centers and many colleges around the world have been established for this purpose. In Islamic teachings, the establishment of contact with God and his memorial has been mentioned as a factor of psychological openness and exaltation which brings many psychological, health effects, religious and Islamic teachings in particular affect the health of individuals [6]. Since Islam can satisfy the need for peace and well-being in mankind and free man from everyday tension. It is Allah's reminder that Allameh Tabatabai explained this issue in this way that the human endeavor is to achieve happiness and well-being and safety from misery. In this way, human grabs any means he has access to but all these things if they are in one direction dominant and capable they are defeated and unable to succeed in the other except Allah the Exalted who is absolute and incapability is not one of his properties [7]. In addition, spirituality is a human and universal experience despite the fact that religious practices and attitudes toward God may vary among different cultures. However, the tendency of humans to communicate with the great and holiest reality over time has been a constant force [8]. Religious and spiritual phenomena/subjects include spiritual and religious way of life, belief and belief in supernatural and subliminal affairs, practice and behavior of spiritual and spiritual beliefs, internal-external religious behaviors, religious or spiritual identity, religious obligations; prayer, forgiveness, remembrance, meditation, presence in tribal gatherings, trust, repentance, recitement, Quran, sacrament, happiness, thanksgiving, fasting, God, death, miracle, angel, soul, peace, humanity, justice, commitment, responsibility and dozens of subjects and other religious and spiritual behaviors which has a direct or indirect relationship with health and disease that has grown dramatically over the past two centuries and have not played a role in providing human health or health care professionals have been neglected [9]. Studies have shown that faith, religious practices, and participation in religious ceremonies can play an effective role in preventing people's psychological problems and their treatment and promote the health and well-being of individuals [10]. The effect of this is perhaps due to the fact that a person becomes self-controlled by increasing religious orientation which prevents the effectiveness of extraterritorial conditions thereby less influenced by inappropriate conditions and preserves his psychological well-being [11]. Yong believed that religion is rooted in the unconscious mind of man, and it strengthens confidence and hope in strength and morale and spirituality in it, creating a strong base against problems, misery and deprivations of life [12]. Also, the reality approach emphasizes that unhealthy communication is the result of unfavorable behavior. This approach emphasizes the focus 
of the authorities on their behavior and refuses to blame the authorities. On the other hand, social well-being as one of the most important subsets of welfare and health includes social communication, social participation, social networks and social support. The feeling of having a social role and identity is one of the important aspects of social welfare. Glaser's Reality Therapy is one of the common therapeutic interventions in the field of psychology in describing human beings, determining behavioral rules and the way to achieve satisfaction, happiness, and success. In this way, the treatment emphasizes on facing reality, accepting responsibility, recognizing basic needs, moral judgment about the correctness or inappropriate behavior, focusing on here and now, internal control and, as a result, achievement of identity that directly relates to self-esteem [13]. Also, the training of reality which is considered as the newest approach to counseling and psychotherapy is based on the theory of choice and control and the cause of psychological problems in choices of individuals and lack of responsibility of the individual to meet their needs in this treatment individuals are better placed to meet their basic needs through better choices and lead to health and well-being in individuals. In fact, the main purpose of this approach is to help individuals to be aware of their needs to monitor behavior and to make appropriate choices. Therapists argue that the underlying problem of most treatment clients is the lack of satisfactory or successful relationships when interacting with people they need in life [14]. Real-life training is a method based on common sense and emotional conflicts in which emphasis is placed upon reality, on the acceptance of responsibility and on the recognition of the right and wrong things and their relationship with the daily life of the individual. Glaser's approach is a unique blend of existential philosophy and behavioral modes that are similar to self-governing behavior of demagogues [15]. Since behaviors have been chosen as a way to solve the frustration caused by an unpleasant relationship then we need to make more selective choices when interacting with those who need them in our lives [16]. Adoption of the fundamentals of choice theory means that we are responsible for all our behaviors. The notion which a person is responsible for his/her behavior is one of the achievements of election theory and when this person accepts an obligation in a position, this responsibility increases [17]. Several studies have shown that the reality of therapy has been effective in multicultural societies [18]. The study of Farahbakhsh and Ghanbari Hashemabadi which entitled as effectiveness of Glaser's couple therapy on post-traumatic stress among betrayal wife showed that reality therapy was effective in reducing stress [19]. The results of Esmali's research and colleagues regarding the effectiveness of reality group therapy on the happiness and quality of life of poorly adolescents in Mashhad showed that reality group therapy is effective in improving the quality of life, increasing happiness and satisfying the lives of poor adolescents; also the respectful environment, emotional expression and empathic understanding in the experimental group are effective in increasing happiness and improving the quality of life [20]. Several studies such as Bilodeau, Jackson, and Wubbolding and Brickells have shown that the reality of therapy is effective on emotional well-being [21-23]. The studies conducted in different parts of the world show the effectiveness of the reality of therapy on a variety of components, problems and psychological disorders such as increasing happiness [24], reducing women's stress and anxiety [25], self-esteem [26], happiness and mental health; general health, life expectancy and anxiety [27].

The study of Haditabar et al showed that spiritual intelligence training has positive effect on psychological well-being of veterans' wives [28]. The research of Asadollahi et al showed that spirituality therapy can be used as an effective therapeutic approach to reduce the frequency of physical symptoms in patients with IBS [29]. Ajdari Fard et al in their research concluded that the training 
of mysticism and spirituality is effective in increasing mental health and improving physical function and social function, and decreasing the anxiety and depression of students [30]. Mar'ashi et al research showed the effect of spiritual intelligence training on reducing existential anxiety and increasing psychological well-being [31]. The research of Heydari et al did not show relationship between spiritual intelligence and happiness through psychological well-being which suggested that spiritual intelligence is a positive predictor of psychological well-being and happiness and also there is a positive and significant relationship between spiritual intelligence and happiness and psychological well-being [32]. Therefore, it is necessary to get acquainted with appropriate treatments such as spiritual education and factual education in group in order to design appropriate measures, to promote mental health services and to create related policies in society. Having information about these two methods can help the student to perform psychological processes so that they can improve their well-being in these people and also raise their life expectancy in them and even the community and also, because spirituality and participation in the group are effective in creating a sense of sympathy and prevention of loneliness in individuals, the researcher consider to usetechniques of this method to examine their impact on the increase of well-being among students. Meanwhile, the results of this research can also be used for universities so that they can pay more attention to train of spirituality, education of reality and psychological well-being by the use of experienced personnel. Therefore the purpose of this study is to compare the effectiveness of reality training and spirituality training on improving psychological well-being among students in Ghorveh.

\section{Method}

This semi-experimental research is a pretest, posttest with control group. The statistical population of this study is all female and male students of different majors of science at Payame Noor University of Ghorveh in the 2015-2016 academic years. In this research, 45 students were selected by sampling method as sample size. The participants were randomly divided into three groups (two experimental and one control group), a test group consisted of 15 students who participated in 9 sessions of spiritual training sessions and participated in spirituality sessions, the second group of the trial consisted of 15 students who underwent 9 sessions of 90 minutes of reality education (based on Glaser's approach) and the control group included 15 students who did not receive any interventions. The meetings were held for three months at the Payame Noor University of Ghorveh. Descriptive statistics and inferential statistics (covariance analysis) were used to analyze the data with SPSS-21 software.

The psychological well-being questionnaire was developed by Carroll Ryff in 1989. This test includes 84 questions and 6 factors. Participants respond to questions at a 6-point scale (totally disagree to fully agree). Forty seven questions, direct, and thirty seven questions are scored invert. Therefore, the above mentioned tool was considered as an effective tool [7]. Cronbach's alpha was obtained from Ryff's study for self-acceptance (0.93), positive relationship with others (0.91), autonomy (0.86), environmental mastery (0.9), purposeful life (0.88) and personal growth (0.87). The reliability coefficient was 0.82 using a re-test of psychological wellbeing scale and self-acceptance subscales, positive relationships with others, autonomy, environmental mastery, purposefulness in life, and personal development were reported $0.71,0.77,0.78,0.90,0.70,0.78$ respectively [33]. The reliability coefficient in the present study was 0.83 with Cronbach's alpha. Summary of the spirituality sessions

- First Session: Preparation, setting goals and rules of the group, familiarizing the members of the group with each other, consulting and discussing spirituality and components of psychological well-being, and implementing a psychological well-being questionnaire.

- Second Session: The story of Joseph and 
God's Wisdom about this story.

- Third Session: Du'a Kumayl and invocation, religious worship.

- Fourth Session: Quran verses and commentary, mentioning of divine names, explanations of divine justice and belief, believing that superior force than us can guide us to the path of health. - Fifth Session: Explaining benefits of participating in religious ceremonies, forgiveness and repentance and divine creativity, believing that Creator is the ally.

- Sixth Session: Listening to the sound of the Holy Quran, and reading the Holy Quran by members of the group.

- Seventh Session: Quran tale and recourse to Ahl-Bayt.

- Eighth Session: Explaining and explaining the benefits of prayer and invocation, the biography of Prophet Muhammad (PBUH).

- Ninth Session: Summarizing and concluding sessions, talking about their learning in the group, reviewing the program and summarizing the curriculum of spiritual therapy, and implementing a psychological well-being questionnaire.

Summary of Reality Sessions (Glasser)

- First session: Familiarity of the members of the group with each other on the training of reality and components of psychological wellbeing and implementation of the psychological well-being questionnaire.

- Second Session: Determining the goals and rules of the group as well as the choice theory.

- Third session: Familiarity of group members with their responsibility and their familiarity with the importance and necessity of responsibility in life; creating a spirit of cooperation with teamwork.

- Fourth Session: The introduction of five basic human needs, such as love and belonging, the need for freedom, the need for strength and progress, the need for recreation, and the physiological need for survival.

- Fifth Session: Familiarity of group members with the basic needs of real life and the impact of basic needs in life and their ability to choose and the best way to meet the basic needs.

- Sixth Session: Group members' familiarity with the quality of planning and solving problems in the current life and choosing better situations.

- Seventh Session: Familiarity with the theory of choice and its role in personal behaviors, general concepts and elements of behavior (the concept of overall behavior), connecting behaviors, creating a healthy morale for establishing good relationships and attachment.

- Eighth Session: Conflict resolution, ability to deal with inconsistency and incompatibility, and find alternative activities, destructive behaviors.

- Ninth Session: Completing and posttest execution.

\section{Results}

The sample size consisted of 23 female and 22 male (41 participants were single and 4 participants married) with an average age of 21 years with a range of 19 to 23 years old.

\begin{tabular}{|c|c|c|c|}
\hline Treatment & Test & Mean & Standard deviation \\
\hline \multirow{2}{*}{ Spirituality } & Pretest & 310.33 & 9.55 \\
\hline & Posttest & 323.01 & 8.97 \\
\hline \multirow{2}{*}{ Reality } & Pretest & 308.01 & 8.37 \\
\hline & Posttest & 317.73 & 8.99 \\
\hline \multirow{2}{*}{ Control } & Pretest & 309.93 & 8.84 \\
\hline & Posttest & 310.47 & 8.96 \\
\hline
\end{tabular}

Based on the data in Table 1, mean and standard deviation, pretest and posttest related to psychological well-being related to spiritual education, faculty education and control group have been shown.
Table 2 shows that the homogeneity of slopes with $F(2.39)=1.72$ for psychological well-being is not meaningful hence the homogeneity assumption of regression slopes for the psychological well-being variable has 
been established. Also, the Levene's variance homogeneity test in the psychological wellbeing variable $(\mathrm{p}=0.69$ and $\mathrm{F}=0.37)$ is not significant at the level of 0.05 . Therefore, the variance of the two groups of experimental and control in the psychological well-being variable is not significantly different and the assumption of the homogeneity of the variances is confirmed. According to the results of Table 2 and Levene's test, covariance analysis was used to study the hypothesis.

Table 2 The results of the test report assume homogeneity of slopes

\begin{tabular}{lcccccc}
\hline Variables & Sum of squares & df & Mean of squares & F & Sig & Square of ETA \\
\hline Group & 8.59 & 2 & 4.3 & 3.88 & 0.029 & 0.166 \\
Pretest & 3338.11 & 1 & 3338.11 & 3017.17 & 0.001 & 0.98 \\
Pretest Group & 3.8 & 2 & 1.9 & 1.72 & 0.192 & 0.08 \\
Error & 43.15 & 39 & 1.11 & - & - & - \\
\hline
\end{tabular}

Table 3. The results of the analysis of covariance in psychological well-being

\begin{tabular}{lccccccc}
\hline Variables & Source of changes & Sum of squares & df & Mean of squares & F & Sig & Square of ETA \\
\hline \multirow{2}{*}{ Psychological } & Pretest & 3337.71 & 1 & 3337.71 & 2914.37 & 0.001 & 0.98 \\
well-being & Group & 1199.87 & 2 & 599.94 & 523.84 & 0.001 & 0.96 \\
& Error & 46.95 & 41 & 1.145 & - & - & - \\
\hline
\end{tabular}

The Table 3 shows that the value of $\mathrm{F}$ $(\mathrm{F}=523.84)$ and the significant level (0.001) in the variable of the group indicate the difference between the two groups of test and control in the posttest. Therefore, the results indicate that spirituality and reality education are effective in increasing the psychological well-being of the participants.
In other words, the training of both spirituality and reality education increased the scores of psychological well-being in the experimental group. It means they have promoted the psychological well-being of students. The value of ${ }^{2} \eta=0.96$ is also a reason for the high effectiveness of these two methods on mentioned variable.

Table 4 Results of moderated averages for psychological well-being

\begin{tabular}{lccccc}
\hline \multirow{2}{*}{ Variable } & \multirow{2}{*}{ Group } & \multirow{2}{*}{ Mean } & \multirow{2}{*}{ Standard deviation } & \multicolumn{2}{c}{$95 \%$ confidence interval } \\
\cline { 5 - 6 } Psychological & Reality & 319.102 & 0.277 & 318.542 & 319.663 \\
well-being & Spirituality & 322.123 & 0.277 & 321.564 & 322.682 \\
& Control & 309.975 & 0.276 & 309.416 & 310.533 \\
\hline
\end{tabular}

Table 4 shows that there is a significant difference in posttest psychological well-being scores after the pretest modulation between the reality groups, the spiritual education and the control group. This means that there is a significant difference between the two groups of posttest scores. Meanwhile, the moderated means of the psychological wellbeing variable are shown in Table 4 . The meaning of the difference in the analysis of covariance does not determine which groups are different. Therefore, a Bonferroni post hoc test was followed, the results of which are shown in Table 5.

Table 5 Bonferroni post hoc test results for comparison of mean scores of psychological well-being of the three groups

\begin{tabular}{llllrr}
\hline \multirow{2}{*}{ Dependent variable } & \multicolumn{2}{c}{ Comparison groups } & \multirow{2}{*}{ Standard deviation } & \multirow{2}{*}{ Standard error } & \multirow{2}{*}{ Sig } \\
\cline { 2 - 5 } & Group & Spirituality & -3.021 & 0.393 & 0.001 \\
\multirow{3}{*}{\begin{tabular}{l}
\multirow{2}{*}{ Psychological well- } \\
being
\end{tabular}} & \multirow{2}{*}{ Training of reality } & Control & 9.128 & 0.392 & 0.001 \\
\cline { 2 - 6 } & \multirow{2}{*}{ Training of spirituality } & Reality & 3.021 & 0.393 & 0.001 \\
& & Control & 12.148 & 0.391 & 0.001 \\
\hline
\end{tabular}

The contents of Table 5, derived from Bonferroni's post hoc test show that there is a significant difference between posttest psychological well-being scores of the reality training and spirituality training Department with the scores of the control group and also, 
there is a significant difference between the posttest scores of psychological well-being in the two interventional groups so that the training of spirituality more than the reality of the treatment increased the psychological well-being. It means training spirituality in comparison of psychological well-being is more effective than training reality.

\section{Discussion}

According to the findings of this research, it can be concluded that spiritual education and reality education are effective in promoting the psychological well-being of students and the training of spirituality to the training of reality is more effective in promoting the psychological well-being of students which is consistent with the other researches [24-32].

In explaining these findings, it can be said that repeated recitation of God produces a productive effect in the human's soul and leads to the excellence of man. Imam Ali said: "whoever keeps his heart by the constant remembrance of God, $\mathrm{He}$ will hold all his actions good in secret and apparent" [34]. This hadith can be a complete and comprehensive explanation for these findings. Participating in religious and worship ceremonies such as prayer reduces tension and escapes emotional distress [35] which can lead to well-being and given the cultural values that govern society and the belief in people's belief in spiritual and religious issues and family patterns, the greater impact of spirituality seems obvious and the results of this research emphasize the claim of the elders and given that the people of our society are inherent in the belief and belief in spiritual matters and consider the spirituality as an integral part of their lives and feel these calm and security issues and even this belief may exist in the collective subconscious, therefore, as a result of this and the greater impact of spirituality that it seems quite natural. As in all religions, recourse to God is common with prayers and verses; in Islam it is also believed that spirituality, the mention of divine names, and Quran verses calms the heart and is effective in curing diseases and it goes without saying that healing is in the interest of divine and the degree of sincerity and human faith. In fact, the training of spirituality promotes calmness of the heart, planning to achieve goals and positive attitudes towards oneself and society to increase hope, well-being, and psychological well-being and all of these components and overall psychological wellbeing can enhance the quality of life, mental health, and thus empowerment of students as human resources. Today, attention to the spiritual dimension of health has been taken into account in many of the definitions presented for health and suggestions for the inclusion of spiritual health as one of the dimensions of health in the definition of the World Health Organization [36].

In addition, focusing on the dimension of responsibility is the core of the work of reality training. Therefore, reality can be effective in learning responsible behaviors in a person and thus preventing anxiety in a person. Specifically, the therapist's reality concentrates all his efforts on behavior, creates a private and active relationship in a responsible manner, and the present, especially the current behavior of references is always intended to succeed. The therapist does not spend time devoting himself to the role of the workshop and the searcher and hears the accusations of the authorities but tries to focus on the behavior of a person more easily than threatened, establishes the basis for responsible behavior and successful health and by reducing the anxiety and intimidation of the personality can be strengthened by this [37] which can explain the impact of reality education on the well-being of students. According to Glaser, group counseling creates an enabling environment that satisfies the needs of the members and increases the responsibility for successful identity which in turn reduces the members' anxiety tension and improves their performance [38]. Satisfying the need for loving and loving ones in the group is well done, and group counseling can provide a good environment for a successful identity and a sense of calm [18]. On the other 
hand, according to Seligman; the creation of a sense of control in individuals, a sense of future, a clear planning, and a sense of power in doing things reduces anxiety [1], this situation can also be a comprehensive and comprehensive explanation for the promotion of psychological well-being. Ultimately, the promotion of wellbeing leads to a positive person's perception of the community and social acceptance, and also social acceptance refers to how much the person feels good to others and how much others feel honestly and persevering. Social acceptance represents an individual's perception of the characteristics and traits of the community as a whole. People who have good levels of this well-being dimension have a positive view of human nature trust others and believe that people can be good, overall, social acceptance means having a positive view of the nature of the human being and being comfortable with others that can lead to the promotion of individuals at all stages of life and the presence of these people leads to the good of the community [39]. Due to the fact that emotions are most interconnected with happiness and well-being, planning can be done in the emotional dimension to a degree that is niche for students. Therefore, the creation and promotion of Islamic culture (trust, honesty, responsibility, etc.) will be effective among students in realizing and increasing the wellbeing. Therefore, the universities contribute to the spiritual well-being of students, as a result of which they will have a double educational achievement and students with more satisfaction and more positive creativity can strive for their scientific and spiritual progress. As a result, the more students will benefit from the level of cognition, emotions and spiritual well-being; they will achieve a more sustainable well-being [40].

The limitation of this research is that considering the fact that the city of Ghorveh has taken place, it should be cautious in generalizing these findings. Therefore, it is recommended for relevant authorities to identify religious values and religious beliefs use the principled, scientific, logical, and psychological features of individuals. The results of this study can be applied to clinical students, psychologists of counseling environments, students, student affairs departments of universities, student deputy of the Ministry of Science, Research and Technology of all university students. Also it is suggested to compare this approach with other ones and survey the effect of this intervention on other variables such as happiness, self-esteem, subjective well-being, self-efficacy and etc.

\section{Conclusion}

According to this matter that spirituality can be trained and acquired, it is suggested that by identifying the factors influencing the training of spirituality and psychological well-being, as well as their limitations, it can be arranged and formulated in a smart manner and provide a platform to help students to grow healthier and more healthy. Obviously, given the importance of the role of spirituality in fostering talent and the training of efficient student forces to build a bright and successful future, the study of spirituality education in students and how it affects psychological well-being, happiness, and emotional intelligence. Any research in this regard is of particular importance. The fact and the choice theory, before being a therapeutic approach is a philosophy and way of life that everyone can take and consider the fact that the method of medical reality is compatible with Iranian culture and the combinations of Islamic religion with emphasis on accountability and reasonableness and simplicity of concepts, this therapeutic approach and that means there is no limitation of the educational orientation for educating therapists in therapeutic reality is a comprehensive approach between different therapeutic approaches to psychology, however having these positive qualities does not have comprehensive and effective spirituality. Considering the culture and beliefs of the Islamic-Iranian society, we need to use and training spirituality in all aspects of life and in 
all stages of life and the effect of this approach towards other approaches and spiritual training is inevitable. Therefore, it is recommended for relevant authorities to identify religious values and religious beliefs use the principled, scientific, logical, and psychological features of individuals. The results of this study can be applied to clinical students, psychologists of counseling environments, students, student affairs departments of universities, student deputy of the Ministry of Science, Research and Technology of all university students.

\section{Acknowledgments}

The researcher is required to thank and appreciate the scholarship and thanks to the cooperation of university officials and students and all the people who have collaborated, answered the questionnaires and participated in the meetings.

\section{Contribution}

Study Design: FNS

Data collection and analysis: FNS

Manuscript preparation: FNS

\section{Conflict of Interest}

The author declares that they have no competing interests.

\section{Funding}

The author received no financial support for this research.

\section{References}

1- Seligman APM, Rauich K, Gecax L, Gilham G. Optimistic child. Tehran: Development publications; 2004.

2- Aghababaei N, Farahani H. The role of a magnitude trap in predicting psychological and factional wellbeing. Journal of Evolutionary Psychology: Iranian Psychologists2011; 8(29): 75-85.

3- Ryff CD, Keyes CL. The structure of psychological wellbeing revisited. Send to. J Pers Soc Psychol1995; 69: 719-27.

4- Vazquez C, Gonzalo H, Juan José R, Diego G. Psychological well-being and health. Contributions of positive psychology. Annuary Clin Health Psychol2009; 5: $15-27$.

5- Myers DG, Diener E. Who is happy? Psychol Sci1995;
6: $10-9$.

6- Khaledian M, Pishvaei M, Karami Baghteyfouni Z, Smaeili M. Effect of Islamic-based spiritual therapy on self-esteem and mental health of addicts. Journal of Research \& Health2017; 7(2): 719-28.

7- Arabi Bafrani H, Kajbaf MB, Abedi A, Habibollahi S. The effectiveness of training of spirituality on married couples' adaptation. Journal of Islamic Studies and Psychology2013; 7(12): 95-112.

8- Hashemi L, Jokar B. The study of the relationship between spiritual excellence and resilience in university students. Psychological Studies of Education2011; 13: 123-42.

9- Bolhari J. Institutionalizing spirituality in the concept of spiritual health. J Med Ethics2010; 4(14): 105-11.

10- Hills P, Francis LJ. Primary personality trait correlates religious practice and orientation. Pers Individ Dif2004; 36: 61-73.

11- Tabraee R, Fathi Ashyani A, Rasoulzade Tabatabaee $\mathrm{K}$. Investigating the contribution of religious orientation on mental health compared to demographic factors. Journal of Psychology and Religion2008; 1(3): 37-62.

12- Zahed Babelan A. Depending on God and the resilience of the meaning of the relationship between the students. Journal of Applied Psychology2012; 13(3): 75-85.

13- Caterin LC. The effect of reality therapy based group counseling on the self-esteem; 2008.

14- Kakia L. Effect of group counseling based on reality therapy on identity crisis in students of guidance schools. J Fundament Mental Health2010; 1:430-7.

15- Prochasca J, Norkras, J. Theories of psychotherapy. Translation by Seyyed Mohammadi. Tehran: Ravan publication; 2017.

16- Soltani Shal R, Aghamohammadian She'rbaf H, Karshki $H$. The role of personality type $D$ and emotional intelligence through perceived stress mediation and coping strategies in the quality of life of heart patients. Monthly Journal of Kermanshah University of Medical Sciences2013; 17: 449-59.

17- Hormozi L, Sepehri Shamloo Z, Alipour A, Zare'a H. Effectiveness of group reality therapy on coping strategies. Journal of Family Psychology2014; 1(2): 19-30.

18- Wubbolding RE, Brickell J, Imhof L, In-za Kim R, Lojk L, Al-Rashidi B. Reality therapy: A global perspective. Int $J$ Advancement of Counselling2004; 26(3): 219-28.

19- Farahbakhsh K, Ghanbari Hashemabadi BA. The study of the effectiveness of Glaser's couple therapy on reducing stress and increasing the severity of posttraumatic consequence of perceptions of betrayal to the spouse. Journal of Clinical Psychology Research and Advice Firdausi University of Mashhad2006; 7(2): 
173-90.

20- Eslami R, Hashemiyan P, Jarahi L, Modares Gharavi $M$. The effectiveness of group therapeutic reality approach on happiness and quality of life in bad adolescents in Mashhad. Journal of Mashhad University of Medical Sciences2013; 56-(5): 300-6.

21- Bilodeau S. Project: impact reality therapy. International Journal of Choice Theory and Reality Therapy2010; 29(2): 69-77.

22- Jackson D. Reality therapy counselors using spiritual interventions in therap. International Journal of Choice Theory and Reality Therapy2014; 33(2): 72-82.

23- Wubbolding RE, Brickell J. The work of brandi roth. International Journal of Choice Theory and Reality Therapy2014; 33(2): 7-11.

24- Mollagholamali Hakak N. Effectiveness of group reality therapy in increasing the students' happiness. Life Science Journal2013; 10(1): 577-80.

25- Al-Sawalma Aisha, Al-Samadi A. The effectiveness of actual treatment in reducing mental stress and anxiety of pregnant women. Jordanian Journal of Educational Sciences 2012; 8(4): 365-76.

26- Moradi Shahr Babak F, Ghanbari Hashem Abadi BA, Agha Mohammadian Shahrbaf H. Effectiveness of group therapy reality therapy on increasing self-esteem of Firdausi university students in Mashhad. Educational Studies and Psychology2010; 11(2): 238-27.

27- Asmakhani Akbari Nejad H, Etemadi A, Nasirnejad F. The effectiveness of group-based therapeutic reality on women's anxiety. Journal of Psychological Studies2014; 10(2): 87-73.

28- Haditabar H, Nawabinajad S, Ahqar, G. The effect of training of spiritual intelligence components on quality of life of veterans' wives working in adult schools in Tehran during the 2010-2011 years. Journal of Veterinary Medicine2011; 4(14): 1-11.

29- Asadollahi F, Neshat Doost H, Kalantari M, Mehrabi H, Afshar H, Doqaghzadeh H. The effect of therapeutic spirituality on physical symptoms in female patients with irritable bowel syndrome. Journal of Behavioral Sciences
Research2014; 12(2): 317-27.

30- Ajdari Fard PS, Ghazi Q, Nooranipour R. The effect of sufism and spirituality training on students' mental health. Journal of New Thoughts in Educational Sciences2010; 5(2): 106-27.

31- Mar'ashi SA, Na'ami A, Bashlida K, Zargar Y, Ghobari Bonab B. The effect of spiritual intelligence training on psychological well-being, existential anxiety, and spiritual intelligence among Ahwaz Oil students. Journal of Psychological Achievements2012; 19(1): 6380.

32- Heydari A, Kouroshniya M, Hosseini Seyedeh M. The relationship between spiritual intelligence and happiness through psychological well-being. Journal of Methods and Psychological Models2015; 6(21): 73-85.

33- Bayani AA, Mohammad Kuchak A, Bayani A. Validity and reliability of Ryff psychological well-being scale. Journal of Psychiatry and Psychology2008; 14(2): 146-51.

34- Leisy Vasseti. Eyes of governance and preaching. Qom : Darolhadith Publications;1997

35- Gholami A, Kiumars B. Effectiveness of spiritual therapy on the mental health of divorced women. Counseling and Psychotherapy Family2011; 1(3): 331-48.

36- Esfahani M. Spirtual health and comments. J Med Ethics2010; 14(4): 41- 9.

37- Shafie Abadi A, Naseri, G. Theories of counseling and psychotherapy. Tehran: University publication center; 2011.

38- Glasser W. Choice theory. New York: Harper collins; 1998.

39- Aghayossefi AR, Fathi A, Shtiani A, Ali Akbari M, Imani Far HR. The effectiveness of the treatmentbased approach based on selection theory on marital conflict and social well-being. Journal of Women and Society2016; 7(2): 1-19.

40- Mozafarinia F, Amin Shokravi F, Heidarnia A. The relationship between spiritual health and happiness in students. Journal of Health Education and Promotion of Health2014; 2(2): 97-107.

Copyright $(0) 2016$ ASP Ins. This open-access article is published under the terms of the Creative Commons Attribution-NonCommercial 4.0 International License which permits Share (copy and redistribute the material in any medium or format) and Adapt (remix, transform, and build upon the material) under the Attribution-NonCommercial terms. 